\title{
Time dilation and the star formation scenario
}

\author{
M. Deng and B.E. Schaefer \\ Department of Physics, Yale University, New Haven, CT 06520-8120, U.S.A.
}

Received December 8, 1998; accepted June 16, 1999

\begin{abstract}
We investigate the dependence of the redshift vs. brightness relation of GRBs upon the width of GRB luminosity function and the cosmological models. The relation is then fit to our observed time dilation data of GRBs. It is found that the best fit average luminosity ranges from $L_{0}=1.1 \pm 0.310^{57}$ photons $\mathrm{s}^{-1}$ in a standard candle luminosity model to $\langle L\rangle=8.7 \pm 3.610^{57}{\text { photons } \mathrm{s}^{-1}}^{-1}$ in a cosmological model with a broad luminosity function $\phi(L)=C \cdot L^{-\beta}$. Our fits suggest that it is only possible to accomodate the large distance scale suggested by the Star Formation Rate model when the luminosity function has a large width $K \sim 1000$ in a cosmological model with low matter density $\Omega_{0}$.
\end{abstract}

Key words: gamma-rays: bursts

\section{Introduction}

Recent advances in optical counterpart observations have greatly reduced the uncertainties in the distance to Gamma Ray Bursts(GRBs). Based on some theoretical models, the GRBs are produced at the end of the life time of massive stars. Since the life time of the massive stars is relatively small, it is argued (Totani 1997, 1998) that the GRB density should be similar to that of the star formation rate (SFR). This model is plausible as it puts the GRBs at larger distance scales $z_{\text {dim }}=5 \sim 6$ (Wijers 1997) than the redshift of $z \sim 1$ for the dim bursts in the conventional model, thus solving the problem that there is a lack of normal galaxies in the error boxes if they are at redshift of $z \sim 1$ (Schaefer 1997).

Such a scenario can be confronted directly with the experimental evidence at hand. It is unclear to what extent this model is supported by the recent determination of the redshifts of a few GRB counterparts, GRB 970508 at $0.85<z<2.1$ (Metzger 1997), GRB 971214 at $z \sim 3.4$ (Kulkarni 1998) and GRB 980703 at $z \sim 0.966$ (Djorgovski 1998). Two of these apparently faint events have host galaxies with $R \sim 25$ mag (Castro-Tirado 1997;
Kulkarni 1998), while the third is similar in brightness but have a bright host galaxy with $R \sim 22 \mathrm{mag}$, therefore GRBs might have a broad luminosity function.

An alternative approach will be using other indirect methods of distance measurement to determine the distance to GRBs and hence the intrinsic luminosity. Totani (1998) examined the GRB number vs. brightness relation and attempted to constrain the GRB density rate to probe the star formation history. However, when the standard candle luminosity assumption is relaxed (Krumholz 1998) and an intrinsic luminosity function is introduced, it is found that the GRB number vs. peak flux relation can be accommodated by a very broad range of models with either comoving densities or distributions tracing star formation history.

In this paper, we use the observed effect of time dilation (Norris et al. 1995; Deng \& Schaefer 1998) to constrain the distance scale of GRBs and compare the result to that predicted by the SFR models.

\section{Analysis}

The time dilation of GRBs is essentially a measurement of the redshift vs. brightness relations. To use the $1+z$ vs. $P$ relation to constrain the distance scale, the following three effects have to be taken into account: the intrinsic luminosity function of GRBs, correction of the peakflux due to redshift of photons in the detector waveband (similar to the K-correction in the optical measurements of galaxy magnitude), and the uncertainty introduced by the specific cosmological models assumed.

We adopt the standard Friedmann-Lemaitre model assuming a power law luminosity function of GRBs $\phi(L)=$ $C \cdot L^{-\beta}\left(L_{\min }<L<L_{\max }\right)$. If this luminosity function is normalized, there are two remaining free parameters, the average luminosity $\langle L\rangle$ and the width of the luminosity function $K=L_{\max } / L_{\min }$. At each observed peak flux level $P$, the GRBs themselves can be located at a wide range of redshifts because of the above luminosity distribution. Therefore we calculate the corresponding average 


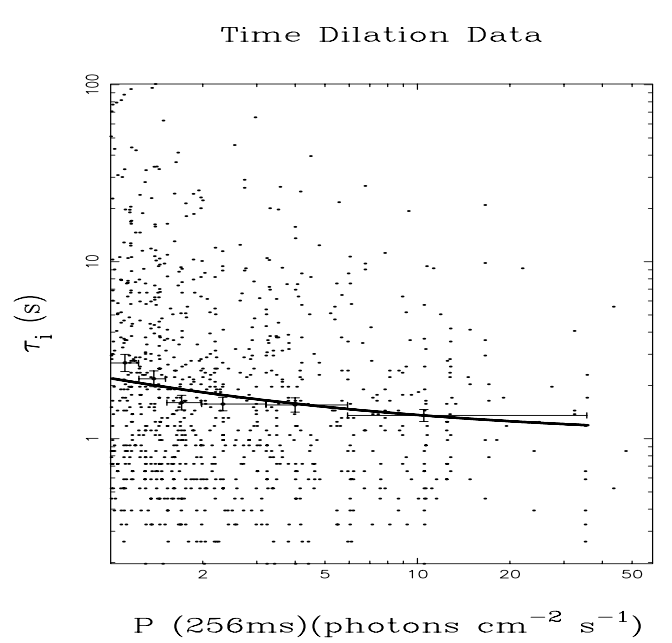

Fig. 1. The time dilation in the peak-to-peak time scales of GRB data with the solid curve being the best fit to a cosmological model with $\Omega_{0}=0.3$ and $\Omega_{\Lambda}=0.7$, a power law luminosity function with $\langle L\rangle=5.4 \pm 2.010^{57}$ photons s $^{-1}$ with $\beta=1.8, K=L_{\max } / L_{\min }=2000.0$ and the spectral index of GRBs $\alpha=2.0$. The unit of peak flux $P$ is photons $\mathrm{s}^{-1} \mathrm{~cm}^{-2}$ from 50 to $300 \mathrm{keV}$ integrated over the $256 \mathrm{~ms}$ data, while the unit of the peak-to-peak time scale $\tau_{i}$ is second

redshift $\langle z\rangle$ of GRBs as sampled from the above luminosity function. When averaging, these redshifts have to be appropriately weighted by the density distribution according to the $\log N-\log P$ curve, and possibly some form of cutoff of the GRB distribution at large redshifts where galaxies are still not formed.

We fit the time dilation data to the above model. The data utilizes the peak-to-peak time scales derived from the time intervals between statistically significant peak structures in the GRB time profiles (Norris et al. 1995; Deng \& Schaefer 1998). It is found that time data of GRBs can be reasonably fit by models with a standard candle luminosity or a broad luminosity function. In the case of models with a luminosity function, the best fit $\langle L\rangle$ is plotted against the change of luminosity function width $K$ as shown in Fig. 2 for a particular model with $\Omega_{0}=0.3$. It is found that the best fit average luminosity rises as the luminosity function width is increased. The cosmological parameters are also varied from the values preferred by the inflationary scenario to the recent values measured by the high redshift supernova surveys.

The combination of the uncertainty in the luminosity function and the cosmological models translates into large variation of the best average luminosity $\langle L\rangle$. The best fit ranges from $L_{0}=1.1 \pm 0.310^{57}$ photons s $^{-1}$ for a cosmological model with $\Omega_{0}=1.0, \Omega_{\Lambda}=0.0$ and a standard candle luminosity $L_{0}$, spectral index $\alpha=2.0$ to as high

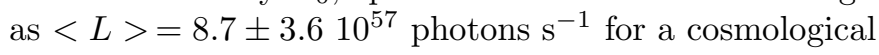
model with $\Omega_{0}=0.3, \Omega_{\Lambda}=0.7$, and a power law luminosity function $\phi(L)=C \cdot L^{-\beta}, \alpha=2.5$.

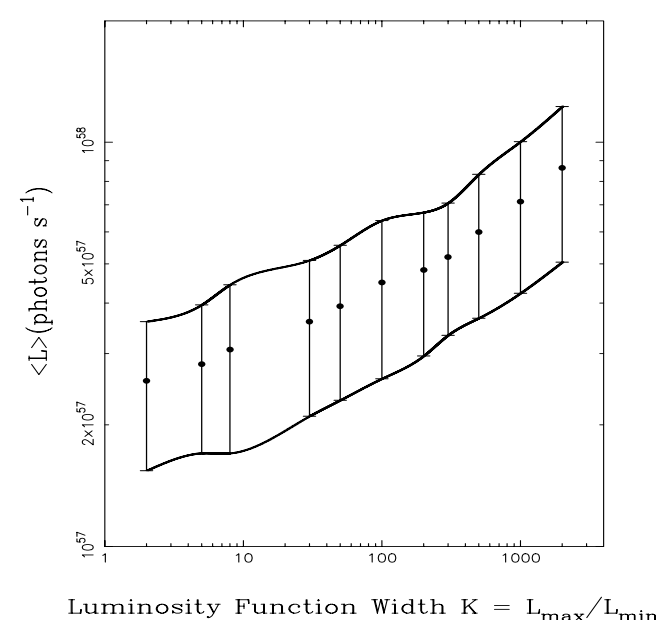

Fig. 2. Best fit average luminosity $\langle L\rangle$ vs. the width $K$ of the luminosity function in a particular cosmological model with $\Omega_{0}=0.3$ and $\Omega_{\Lambda}=0.7$, luminosity function index $\beta=1.8$, and the spectral index of GRBs $\alpha=2.0$. This graph shows that the best fit of $\langle L\rangle$ changes by a factor of $\sim 3$ with a broad luminosity function width $K=2000$

\section{Conclusion}

The uncertainties in the GRB luminosity function and cosmological parameters can easily translate into an order of magnitude uncertainty in using the time dilation or directly measured redshift vs. peak flux relation to constrain the distance scale and luminosity of GRBs. These should be taken into account when one attempts to distinguish between the traditional models with $z \sim 1$ and the SFR models with GRBs at much higher redshifts $z \sim 5-6$ (Wijers 1997) for dim GRBs with peak flux $P \sim 0.3$ photons $\mathrm{s}^{-1} \mathrm{~cm}^{-2}$. For SFR models which require a peak luminosity $L \sim 10^{58}$ photons $\mathrm{s}^{-1}$, the above fits suggest that it is only possible to accomodate such a scenario when the luminosity function has a large width $K \sim 1000$ in a cosmological model with low matter density $\Omega_{0}=0.3$.

\section{References}

Castro-Tirado A.J., 1997, IAU Circ. 6848

Deng M., Schaefer B.E., 1998, ApJ 502, L109

Djorgovski S.G., 1998, ApJL 508, L17

Krumholz M., et al., 1998, ApJL 506, L81

Kulkarni S.R., et al., 1998, Nat 393, 35

Metzger M.R., et al., 1997, Nat 387, 878

Norris J.P., Nemiroff R.J., Bonnel J.T., Scargle J.D., 1995, Gamma-Ray Bursts, Kouveliotou C., Briggs M.S., Fishman G.J. (eds.), AIP Conf. Proc. 384, 72

Schaefer B.E., et al., 1997, ApJL 489, 693

Totani T., 1997, ApJ 486, L71

Totani T., 1998, astro-ph/9801105

Wijers A.M.J.R., Bloom S.J., Bagla S.J., Natarajan P., MNRAS 1, 1997 